\title{
Genotipos de virus papiloma humano (VPH) en pacientes con cáncer cervico-uterino en un hospital público y una clínica privada de Santiago, Chile
}

\author{
Isabel M. Valdivia L., Francisco Aguayo G., Martha Pruyas A., \\ Peter J.F. Snijders, Alejandro Corvalán R. y Catterina Ferreccio R.
}

\section{Human papillomavirus (HPV) genotypes in cervix uterine cancer patients in a public hospital and private clinic from Santiago, Chile}

We compared HPV genotypes among squamous cervical cancer samples from a public hospital $(\mathrm{n}=55)$ and a private clinic $(n=35$ cases) of Santiago. Paraffin-embedded specimens were analyzed by PCR followed by an immunoenzimatic assay. Reverse line blotting was used for the identification of $36 \mathrm{HPV}$ genotypes. We found HPV DNA in 94.4\% of all cancers. Single infections: HPV16: 40.0\%, (clinic 37.1\%, hospital 41.8\%) VPH18:7.8\% (clinic 2.9\%, hospital 10.9\%); single+multiple infections: VPH16: 61.1\% (clinic 53.1\%, hospital 71.7\%), VPH18: $34.4 \%$ (clinic $21.9 \%$, hospital $45.2 \%$ ). HPV16 or HPV18 occurred in $75.6 \%$ of cases, higher in the hospital than the clinic (87.3\%-95\% CI: 84.9-96.3 - and 57.1\%-95\% CI: 46.6-66 - respectively, $\mathrm{p}=0.002)$. Other genotypes in single infections: HPV 26, 31, 33, 45, 58, 67; in co-infections: HPV 35,52,56,59 and 66. HPV16 but specially HPV 18 were significantly more frequent in the public hospital; $75.6 \%$ of squamous cervical cancer were associated to the vaccine preventable HPV16/18.

Key words: Human papillomavirus (HPV), cervical cancer, genotypes.

Palabras clave: Virus papiloma humano (VPH), cáncer cervical, genotipos.

\section{Introducción}

$\mathrm{E}$ 1 cáncer cérvico-uterino $(\mathrm{CaCu})$ es el segundo cáncer más común en mujeres en el mundo y la primera causa de muerte por cáncer en mujeres en países en vías de desarrollo ${ }^{1}$. En Chile, la tasa de mortalidad por esta enfermedad fue de 8,2 cada 100.000 mujeres en el año 2005, siendo una de las principales causas de muerte por cáncer en mujeres jóvenes ${ }^{2}$. El virus papiloma humano (VPH) es la causa necesaria para el desarrollo de esta enfermedad. Se han identificado más de 120 genotipos de VPH, pero sólo algunos de ellos, denominados como VPH de alto riesgo (VPHAR), se asocian con infecciones en el tracto genital y el desarrollo de cáncer cervical ${ }^{3-5}$. Los VPH de bajo riesgo se asocian a lesiones benignas del tracto genital, tales como condilomas ${ }^{6}$. Las vacunas profilácticas contra los genotipos de VPH 16 y 18 (bivalente) y VPH 6, 11, 16 y 18 (tetravalente) han demostrado una alta protección contra infecciones de estos genotipos ${ }^{7,8}$. Por otro lado, se han desarrollado técnicas moleculares para identificar los genotipos más frecuentes en los cánceres invasores en el mundo9 . La más utilizada hasta la fecha es la detección del ADN viral mediante el método de captura de híbridos (hybrid capture) el cual permite identificar a los 13 genotipos de VPH responsables de más de $95 \%$ de los cánceres cérvico-uterinos en el mundo ${ }^{10}$.

La distribución de genotipos de VPH en la población general y en las mujeres con lesiones pre-cancerosas o con cáncer, varía entre países e incluso entre poblaciones del mismo país ${ }^{9,11}$. En Chile, se ha reportado una amplia variación regional en los tipos de VPH en la población general de mujeres ${ }^{12}$. El objetivo de este estudio fue comparar la distribución de los genotipos de VPH en especímenes de $\mathrm{CaCu}$ escamocelular obtenidos de un hospital público y de una clínica privada de Santiago, Chile, donde se atiende preferentemente personas de estrato socioeconómico bajo y medio-alto respectivamente. En Chile, 90\% de la población que se atiende en los hospitales públicos tiene bajos ingresos, cifra que se reduce a menos de $10 \%$ de bajos ingresos en los atendidos en sistemas privados de salud ${ }^{13-15}$.

Este estudio fue aprobado por el Comité de Ética de la Pontificia Universidad Católica de Chile.

\section{Materiales y Métodos}

Muestras clínicas. Se realizó una búsqueda simultánea en las bases de datos del Hospital Sótero del Río (HSR) (que atiende una población de bajo nivel socio-econó-
Pontificia Universidad Católica de Chile, Santiago Centro de Investigaciones Médicas Laboratorio de Patología Molecular y Epidemiología (IMVL, FAG, ACR, CFR)

Departamento de Anatomía Patológica (ACR) Departamento de Salud Pública (FAG, CFR) Hospital Dr. Sótero del Río, Santiago, Chile Departamento de Anatomía Patológica (MPA)

University Medical Center, Amsterdam, Países Bajos Department of Pathology, VU (PJFS)

Este trabajo fue financiado por el proyecto Fondecyt 1060645.

Los autores declaran no tener conflictos de interés

Recibido: 22 de junio de 2009 Aceptado: 27 de octubre de 2009

Correspondencia a: Catterina Ferreccio Readi cferrec@med.puc.cl 
mico) y en aquellas de la Clínica Pontificia Universidad Católica (CPUC) (que atiende una población de nivel socioeconómico medio-alto). Para ingresar al estudio, se requirió que estuvieran disponibles los bloques de parafina con las muestras y las láminas histológicas. Los diagnósticos de $\mathrm{CaCu}$ fueron confirmados por dos experimentados patólogos usando los criterios de la convención de Bethesda. Se logró identificar 55 casos de $\mathrm{CaCu}$ escamocelular en el HSR atendidos entre 2005 y 2006 y 35 casos en la CPUC atendidos entre 1996 y 2002.

Extracción de ADN. Todas las muestras del hospital y de la clínica se procesaron simultáneamente y con los mismos métodos. Con un micrótomo, se obtuvo dos cortes de $8 \mu \mathrm{m}$ de cada muestra incluida en parafina. Para cortar secciones de bloques de parafina se siguió el método descrito por Volkenadt y cols ${ }^{16}$. En breve, se realizó un cambio de cuchillas cada tres bloques, entre cada uno de éstos se limpió la cuchilla con xilol y se desplazó lateralmente de modo que cada bloque enfrentó un nueva parte de la cuchilla. Pronto, todas las cuchillas se colocaron en una bolsa cerrada para evitar contaminación por aerosol. Los cortes se pusieron en un portaobjetos de vidrio y se trataron con xilol por $5 \mathrm{~min}$, etanol al $100 \%$ por $5 \mathrm{~min}$, etanol $95 \%$ por 5 min y etanol $70 \%$ por $3 \mathrm{~min}$; luego se tiñeron con hematoxilina-eosina. Posteriormente se microdisecó manualmente las áreas ricas en células tumorales (área > $70 \%$ ) usando una cuchilla diferente para cada muestra. Los cortes se pusieron en tubos de microcentrífuga de 1,5 $\mathrm{mL}$ para obtener extractos crudos de ADN y se incubaron con $500 \mu \mathrm{l}$ de solución de lisis $(20 \mu \mathrm{g} / \mathrm{ml}$ proteinasa $\mathrm{K}$; $0,01 \mathrm{M}$ EDTA pH 8,0;0,5\% Tween 20;0,02 M Tris $\mathrm{pH}$ $8,0)$ por $48 \mathrm{~h}$ a $37^{\circ} \mathrm{C}$. Luego se incubaron por $10 \mathrm{~min}$ a $100^{\circ} \mathrm{C}$, se centrifugaron y almacenaron a $4^{\circ} \mathrm{C}$ para los análisis posteriores.

Reacción de polimerasa en cadena (RPC). Se llevó a cabo usando los partidores GP5+/6+ para amplificar un fragmento de L1 de VPH (155 bp) ${ }^{17}$.

Ensayos EIA y RLB. Los fragmentos obtenidos a través de la amplificación mediante RPC con los partidores GP5+/6+ se analizaron con un ensayo inmuno-enzimático (EIA) para determinar la presencia de un amplio espectro de genotipos de VPH usando dos cócteles de sondas específicas ${ }^{17}$. Un cóctel contenía sondas para genotipos de alto riesgo $(16,18,26,31,33,35,39,45,51,52,53$, $56,58,59,66,68,67,73$ y 82) y otro cóctel contenía sondas para genotipos de riesgo bajo e indeterminado (6, 11, 34, 40, 42, 43, 44, 54, 61, 70, 71, 72, 81 у CP6108). Los genotipos de VPH se clasificaron en riesgo alto, bajo o indeterminado según revisiones recientes ${ }^{3-5}$. Para genotipificar, los productos de RPC positivos en RPC/ EIA, se usó reverse line blotting (RLB), con métodos previamente descritos ${ }^{18}$. Este ensayo permite detectar los tipos de VPH de bajo riesgo: 6, 11, 34, 40, 42, 43, 44, 54, $55,57,61,70,71,72,81$ у CP6108; VPH alto riesgo: $16,18,26,31,33,35,39,45,51,52,53,56,58,59,66$, 67,68, 69, 73, 82 (subtipo IS39), 82 (subtipo MM4). Además, se usaron partidores específicos para detectar la presencia de los genotipos 16 y 18, mediante RPC, seguido de confirmación de los productos con Southernblot $^{19}$. Cuando los ensayos de RPC para detectar VPH fueron consistentemente negativos, se evaluó la calidad del ADN de las muestras con la detección de un fragmento de $\beta$-globina humana ${ }^{20,21}$. Las muestras positivas para el análisis de RPC/EIA en las que no se detectó un genotipo en el ensayo de RLB se clasificaron como VPHX.

Análisis estadísticos. Se comparó la frecuencia de los diversos genotipos de VPH entre casos del HSR y de la CPUC. La significancia estadística de los resultados se estimó con la prueba exacta de Fisher.

\section{Resultados}

Se completó el estudio de VPH en 90 muestras de cáncer cérvico-uterino escamocelular; 55 provenientes del HSR y 35 de la CPUC. Se aisló algún VPH oncogénico o de alto riesgo (VPHAR) en la mayoría de los cánceres estudiados $(94,4 \%$; 95\% IC = 89,3-98,7) sin diferencias significativas entre la CPUC y el HSR (91,4\% IC 95\%= $82,1-100 \%$ y $94,5 \%$, IC95\% $=90,3-100,0 \%$, respectivamente) (Tabla 1). Los VPH 16 y 18 fueron los genotipos predominantes $(75,5 \%)$ siendo significativamente menos frecuentes en la CPUC que en el HSR $(57,1$ y 87,2\%, respectivamente) (Tabla 1). Los casos tuvieron edades de 24 a 88 años, algo mayores en el HSR que en la CPUC (edad promedio $51 \pm 15$ años y $48 \pm 11$ respectivamente). Los VPH 16 o 18 disminuyeron con la edad, tendencia más evidente en el HSR; sin embargo, este comportamiento no alcanzó significación estadística (Tabla 1).

La Tabla 2 presenta los genotipos de virus aislados. En todos los casos las muestras fueron adecuadas para el estudio, incluso las que fueron negativos para VPH por $\mathrm{RPC} /$ EIA resultaron positivos para el ensayo de RPC para $\beta$-globina humana (prueba de calidad de la muestra) ${ }^{20,21}$. Se logró genotipificar al $100 \%$ de los VPH aislados en el HSR y el $88,6 \%$ de los casos de la CPUC; las muestras no genotipificables resultaron positivas para el cóctel de sondas de tipos de alto riesgo con RPC/EIA pero negativas frente al método de RLB, sugiriendo la presencia de tipos relacionados o subtipos/variantes, siendo designadas AR-HPVX. El VPH18 tuvo el doble de prevalencia en el HSR que en la CPUC (45,2 y 21,9\% respectivamente, $\mathrm{p}=0,03)$, en tanto el HPV16 fue 35\% más frecuente en los casos del hospital $(71,7$ y $53,1 \%$, respectivamente, 


\begin{tabular}{|c|c|c|c|c|}
\hline & $\begin{array}{c}\text { Total } \\
\text { n: } 90(\%)\end{array}$ & $\begin{array}{c}\text { CPUC } \\
\text { n: } 35(\%)\end{array}$ & $\begin{array}{c}\text { HSR } \\
\text { n: } 55 \text { (\%) }\end{array}$ & $\mathbf{P}$ \\
\hline VPH AR - & $5(5,6)$ & $3(8,6)$ & $2(3,6)$ & ns \\
\hline VPH AR + & $85(94,4)$ & $32(91,4)$ & $53(94,5)$ & ns \\
\hline VPH16 ó 18 + & $68(75,5)$ & $20(57,1)$ & $48(87,3)$ & 0,002 \\
\hline Edad en años & $\begin{array}{c}\text { Casos estudiados } \\
\text { (\% VPH AR+) }\end{array}$ & \multicolumn{2}{|c|}{$\begin{array}{c}\text { Casos estudiados } \\
\text { (\% VPH AR +) }\end{array}$} & valor $p$ \\
\hline Total & $90(95,0)$ & $35(91,4)$ & $55(94,5)$ & ns \\
\hline$\leq 39$ & $20(95,2)$ & $9(88,9)$ & $11(100)$ & ns \\
\hline $40-49$ & $37(91,9)$ & $17(100)$ & $20(95,0)$ & ns \\
\hline$\geq 50$ & $33(84,8)$ & $9(77,7)$ & $24(91,7)$ & ns \\
\hline \multirow{2}{*}{ Edad } & VPH $16 / 18+(\%)$ & \multicolumn{2}{|c|}{ VPH $6 / 18+(\%)$} & valor $p$ \\
\hline & $68(75,5 \%)$ & $20(57,1)$ & $45(81,8)$ & 0,01 \\
\hline$\leq 39(n: 21)$ & $16(80,0)$ & $5(55,5)$ & $11(100)$ & 0,04 \\
\hline 40-49 (n: 39) & $17(45,9)$ & $10(58,8)$ & $17(85,0)$ & 0,02 \\
\hline$\geq 50$ (n: 30) & $22(66,6)$ & $5(55,5)$ & $17(70,8)$ & ns \\
\hline
\end{tabular}

$\mathrm{p}=0,08)$. VPH1 8 fue el único tipo en $22,6 \%$ de los casos infectados por este tipo (14,3 y $25 \%$ de los detectados de la CPUC del HSR respectivamente); por el contrario, VPH 16 fue tipo único en $60,5 \%$ de los casos en que se lo detectó $(76,5$ y $60,5 \%$ de los detectados en la CPUC y en el HSR, respectivamente) (Tabla 2) Además de los VPH16 y 18 se identificaron otros 11 genotipos de VPH destacando por frecuencia los VPH 58, 45, 33 y 31. Los que fueron tipos únicos en 11 casos, 12,2\% de los casos totales (20\% en la CPUC y 7,3\% en el HSR) (Tabla 2). No se detectó VPH de bajo riesgo. En 25 casos se detectó más de un genotipo de VPH $(27,8 \%)$, la mayoría de ellas se acompañaba de VPH 16 (76\%) o 18 (96\%).

\section{Discusión}

Este es el primer reporte en Chile sobre variabilidad en los genotipos de VPH asociados a cáncer cérvico-uterino en mujeres atendidas en distintos sistemas de salud asociados a diferentes estratos socio-económicos ${ }^{13-15}$. A pesar de ser un estudio retrospectivo, con muestras archivadas, fue posible detectar el VPH en la gran mayoría de éstas $(94,4 \%)$, genotipificándose también una alta proporción (90\%). Se identificaron diferencias estadísticamente significativas en los genotipos prevalentes en mujeres provenientes de la CPUC en comparación con el HSR. Aunque VPH 16 y 18 son los genotipos más prevalentes en $\mathrm{CaCu}$ y en los tumores relacionados con $\mathrm{VPH}$, su importancia relativa varía ampliamente en el mundo ${ }^{22}$. Algunos reportes sugieren que las diferencias de prevalencia entre casos de $\mathrm{CaCu}$ pueden ser asociadas a factores relacionados con el estrato socio-económico ${ }^{23-25}$.

Un estudio previo recientemente reportado ${ }^{26}$ en 300 muestras chilenas de varios hospitales, encontró las mismas cifras promedio de prevalencia descritas por nosotros: detección de VPH en 94,2\% de los cánceres invasores y VPH 16 ó 18 en $86,8 \%$ de éstos. Sin embargo, ellos no describen la variabilidad entre hospitales.

La heterogeneidad en los tipos de VPH podría ser particularmente relevante en Chile, donde el sistema de salud es dual, con un sistema de salud privado que cubre aproximadamente $30 \%$ de la población (con mayores ingresos económicos), y un sistema de salud público que cubre el $70 \%$ restante de la población (con menores ingresos económicos $)^{27}$. A nivel internacional se ha descrito mayor proporción de VPH 16 y 18 en casos de cáncer cérvico-uterino en Europa Oeste en comparación con áreas menos desarrolladas como América Central $(73,6 \text { y } 58,4 \% \text {, respectivamente })^{28}$. Por ello, se esperaba mayor prevalencia de VPH 16 y 18 en los casos de nivel socioeconómico más alto, correspondientes a aquellos 


\begin{tabular}{|c|c|c|c|c|c|}
\hline \multicolumn{2}{|l|}{ Genotipo de VPH } & $\begin{array}{c}\text { Todos } \\
\text { n: } 90(100 \%)\end{array}$ & \multirow{2}{*}{$\begin{array}{c}\text { CPUC } \\
\text { n: } \mathbf{3 5}(\mathbf{1 0 0 \% )} \\
13(37,1)\end{array}$} & \multirow{2}{*}{$\begin{array}{c}\text { HSR } \\
\text { n: } 55 \text { (100\%) } \\
23(41,8)\end{array}$} & \multirow{2}{*}{$\begin{array}{c}\begin{array}{c}\text { Valor p } \\
\text { CPPUC vs HSR }\end{array} \\
0,83\end{array}$} \\
\hline \multirow{9}{*}{ VPH AR únicos } & 16 & $36(40,0)$ & & & \\
\hline & 18 & $7 \quad(7,8)$ & $1 \quad(2,9)$ & $6(10,9)$ & 0,24 \\
\hline & 26 & $1(1,1)$ & $1 \quad(2,9)$ & $0 \quad(0)$ & 0,39 \\
\hline & 31 & $3(3,3)$ & $1 \quad(2,9)$ & $2(3,6)$ & 1,00 \\
\hline & 33 & $3(3,3)$ & $3(8,6)$ & $0 \quad(0)$ & 0,06 \\
\hline & 45 & $3(3,3)$ & $1 \quad(2,9)$ & $2(3,6)$ & 1,00 \\
\hline & 58 & $2(2,2)$ & $2(5,7)$ & $0 \quad(0)$ & 0,15 \\
\hline & 67 & $1(1,1)$ & $0 \quad(0)$ & $1(1,8)$ & 1,00 \\
\hline & $X(*)$ & $4(4,4)$ & $4(11,4)$ & $0 \quad(0)$ & 0,02 \\
\hline \multirow{9}{*}{ VPH múltiples } & $16 / 18$ & $17(18,9)$ & $4(11,4)$ & $13(23,6)$ & 0,18 \\
\hline & $16 / 58$ & $1(1,1)$ & $0 \quad(0)$ & $1(1,8)$ & 1,00 \\
\hline & $18 / 58$ & $1(1,1)$ & $0 \quad(0)$ & $1 \quad(1,8)$ & 1,00 \\
\hline & $35 / 18$ & $1(1,1)$ & $0 \quad(0)$ & $1(1,8)$ & 1,00 \\
\hline & $52 / 18$ & $1(1,1)$ & $1(2,9)$ & $0 \quad(0)$ & 0,39 \\
\hline & $56 / 18$ & $1(1,1)$ & $0 \quad(0)$ & $1(1,8)$ & 1,00 \\
\hline & $59 / 18$ & $1(1,1)$ & $1 \quad(2,9)$ & $0 \quad(0)$ & 0,39 \\
\hline & $66 / 18$ & $1(1,1)$ & $0 \quad(0)$ & $1(1,8)$ & 1,00 \\
\hline & $16 / 18 / 59$ & $1(1,1)$ & $0 \quad(0)$ & $1(1,8)$ & 1,00 \\
\hline \multirow{3}{*}{ Total VPH 16 ó 18} & 16 & --- & $17(53,1)$ & $38(71,7)$ & 0,08 \\
\hline & 18 & --- & $7(21,9)$ & $24(45,2)$ & 0,03 \\
\hline & 16 ó 18 & $68(75,6)$ & $20(57,1)$ & $48(87,3)$ & 0,002 \\
\hline
\end{tabular}

atendidos en la CPUC, que en mujeres del HSR. En particular, la presencia de VPH 18 fue inesperadamente alta en casos del HSR (43,6\%) comparado a otros países de América, donde la prevalencia de este genotipo en cáncer cervical es de $6,7 \%, 12,5 \%, 14,1 \%$ y $21,6 \%$ en el Caribe, Sudamérica, América Central y Norteamérica, respectivamente ${ }^{28}$. Este puede ser un fenómeno aleatorio o representar efectivamente un mayor prevalencia de este genotipo entre la población residente en el SSMSO. Recientemente reportamos en una cohorte de mujeres de la comuna de La Pintana correspondiente al HSR, estudiadas el 2001 y 2006 , un aumento de $24 \%$ en los anti-cuerpos anti VPH 18 y un aumento de la portación de ADN de VPH 18 de 0,5\% en 2001 a 2,8\% en 2006. Por el contrario, en el mismo estudio, las prevalencias de anticuerpos o ADN viral para VPH 16 prácticamente no cambiaron en el período ${ }^{29.30}$. Esta evidencia, aunque indirecta, apoyaría la hipótesis de una mayor prevalencia de VPH 18 en la población atendida por el Servicio de Salud Metropolitano Sur Oriente. Este tema sólo podrá ser clarificado con nuevos estudios prospectivos, tanto entre las mujeres de la población general como entre aquellas con cáncer invasor residentes en diferentes zonas del país.

En un caso de la CPUC se detectó el genotipo poco común VPH 26. Este genotipo ha sido detectado en casos de cáncer cervical de América del Sur, pero no en Europa, lo que es interesante, considerando que un reporte reciente concluyó que VPH 26 podría ser de riesgo intermedio para el desarrollo de cáncer cervical ${ }^{31}$.

Se detectó co-infecciones en $27,7 \%$ de los casos, un porcentaje mayor que un reporte previo de Temuco ${ }^{32}$ pero prácticamente igual a un reporte de este año en una mues- 
tra grande de varias ciudades de Chile $(27 \%)^{26}$; a nivel mundial las co-infecciones varían entre 9 y $40 \%{ }^{33-37}$. Todas las co-infecciones de nuestra serie presentaban VPH 16 o 18 , similar a lo descrito en reportes internacionales ${ }^{33,34}$. VPH 18 estaba presente en 90\% de las co-infecciones, cifra mayor a la obtenida en estudios previos en Chile que mostraban un $66,7 \%$ de VPH 18 en co-infecciones ${ }^{32}$, y $53,2 \%$ a nivel mundial ${ }^{33}$. Encontramos infección única por VPH 16 en $65,5 \%$ de las infecciones por este virus en comparación con sólo $22,6 \%$ en las infecciones por VPH18. Similar al estudio reciente de Roa y cols ${ }^{26}$, para el caso de VPH16: 65,8\% como infecciones únicas, pero él encontró una tasa mayor de VPH 18 como infección única: $47,9 \%$. El estudio internacional de Muñoz y cols ${ }^{33}$ reporta VPH 16 en infección única 70,4\% VPH18 en 23,2\% $\%^{33}$. muy similar a nuestro hallazgo. Es necesario avanzar a estudios moleculares para clarificar el rol causal de cada uno de estos agentes.

En conclusión, se encontró VPH 16 y 18 en 75,6\% de los cánceres cérvico-uterinos escamocelulares estudiados, variando de 57,1 a $87,2 \%$ entre los casos de la clínica y los del hospital, respectivamente. Estos hallazgos deben ser confirmados en estudios de prevalencia que incorporen un mayor número de establecimientos, tanto públicos como privados, en diversas regiones y donde se pueda recoger prospectivamente mayor información sobre los casos. Una vacuna profiláctica anti VPH 16 y 18 sería, teóricamente, útil en la prevención de lesiones causadas por estos tipos de VPH. Sin embargo, el beneficio potencial de la vacuna podría ser heterogéneo a lo largo del país.

\section{Agradecimientos}

Agradecemos a Tulio Rodríguez por su amable colaboración en la recolección de muestras, a Solana Terrazas por sus comentarios editoriales sobre el manuscrito y la colaboración de laboratorio del Departamento de Patología Molecular de Peter Snijders.

\section{Resumen}

Se comparan los genotipos de VPH en casos de cáncer cérvico-uterino escamocelular de una clínica privada (n: 35) y de un hospital público (n: 55) atendidos entre 1996 y 2006 en Santiago, Chile. Se analizaron por RPC y ensayo inmunoenzimático muestras tumorales en bloques de parafina, genotipificándose con reverse line blotting para 36 genotipos de VPH. Se detectó VPH en 94,4\% de los casos: infecciones únicas por: VPH 16: 40,0\%, (clínica 37,1\%, hospital 41,8\%) VPH 18: 7,8\% (clínica 2,9\%, hospital 10,9\%); total de infecciones por VPH 16 : $61,1 \%$ (clínica $53,1 \%$, hospital $71,7 \%$ ), por VPH 18 : 34,4\% (clínica 21,9\%, hospital 45,2\%). Co-infección: VPH 16/18 75,6\% (clínica 57,1\%; IC95\% = 46,6-66,0 hospital 87,3\%; IC95\% $=84,9-96,3, p=0,002)$. Se identificó otros 11 genotipos oncogénicos en infecciones únicas (VPH: 26, 31, 33, 45, 58, 67) o en co-infección con VPH 16/18 (VPH: 35, 52, 56, 59, 66). VPH 16 y VPH 18 fueron significativamente más frecuentes en el hospital público, particularmente VPH18; 75,6\% de los cánceres se asociaron a los genotipos VPH16/18, tipos prevenibles por vacuna.

\section{Referencias}

1.- Sankaranarayanan R, Ferlay J. Worldwide burden of gynaecological cancer: the size of the problem. Best Pract Res Clin Obstet Gynaecol 2006; 20: 207-25.

2.- Ministerio de Salud Gobierno Chile. Mortalidad por causas; Serie mortalidad por causas; Tumores malignos; Cáncer del cuello uterino. [accedido octubre 2009]. Disponible en: http:// www.minsal.cl/.

3.- Muñoz N, Castellsagué X, de González AB, Gismann L. Chapter 1: HPV in the etiology of human cancer. Vaccine 2006; 24S3: S3/1-10.

4.- IARC Monographs on the evaluation of carcinogenic risks to humans 2007 vol 90 Human papillomaviruses. Disponible en http://monographs.iarc.fr/ENG/Monographs/vol90/index.php, (accedido 12 octubre 2009).

5.- Schiffman M, Herrero R, DeSalle R, Hildesheim A, Wacholder S, Rodriguez A C, et al. The carcinogenicity of human papillomavirus types reflects viral evolution. Virology 2005; 337: 7684.
6.- Gall S A. Female genital warts: global trends and treatments. Infect Dis Obstet Gynecol 2001; 9: $149-54$

7.- Lowy D R, Schiller J T. Prophylactic human papillomavirus vaccines. J Clin Path 2006; 116: 1167-73.

8.- Human papillomavirus (HPV) vaccine background paper. September 2009. Disponible en: http://www.who.int/immunization/documents/ HPVBGpaper05032009.pdf. (accedido octubre 2009).

9.- Quint W G V, Pagliusi S R, Lelie N, de Villiers E M, Wheeler C M. Results of the First World Health Organization International Collaborative Study of Detection of Human Papillomavirus DNA. J Clin Micro 2006; 44: 571-79.

10.- Meijer C, Berkhof J, Castle P E, Hesselink A T, Franco EL, Ronco G, et al. Guidelines for human papillomavirus DNA test requirements for primary cervical cancer screening in women 30 years and older. Int J Cancer 2009; 124: 516-20.

11.- Smith J S, Lindsay L, Hoots B, Keys J, Franceschi S, Winer R, et al. Human papillomavirus type distribution in invasive cervical cancer and high-grade cervical lesions: a meta-analysis update. Int J Cancer 2007; 121: 621-32.

12.- Ferreccio C, Corvalán A, Margozzini $P$, Viviani P, González C, Aguilera X, et al. Baseline assessment of prevalence and geographical distribution of HPV types in Chile using self-collected vaginal samples. BMC Public Health 2008: 8: 78.

13.- Encuesta Casen 2003. Módulo Salud. Disponible en: www.mideplan.cl. (accedido octubre 2009).

14.- Estadísticas. FONASA. Disponible en www. fonasa.cl. (accedido octubre 2009).

15.- Superintendencia de Salud, Diagnóstico del Sistema Isapre, Nov 2005, Disponible en: www. supersalud.cl. (accedido octubre 2009).

16.- Volkenadt M, Dicker A, Fanina R, Banerje D, Albino A, Bertino J. DNA from paraffin-embedded tissue in: Methods in Molecular Biology (volumen 15) - PCR protocols current methods and applications. Bruce A. White (ed) 1993. Human Press, pp 81-8.

17.- de Roda Husman A M, Walboomers J M, van de 
Brule A J, Meijer C J, Snijders P J. The use of general primers GP5 and GP6 elongated at their 3 ' adjacent highly conserved sequences improves human detection by PCR. J Gen Virol 1995; 76: 1057-62.

18.- van den Brule A J, Pol R, Fransen-Daalmeijer N, Schouls L M, Meijer C J, Snijders P J. GP5+/6+ PCR followed by reverse line blot analysis enables rapid and high-throughput identification of human papillomavirus genotypes. J Clin Microbiol 2002; 40: 779-87.

19.- Baay M F D, Quint W G V, Koudstaal J, Hollema H, Duk J M, Burger M P M. Comprehensive study of several general and type-specific primer pairs for detection of human papillomavirus DNA by PCR in paraffinembedded cervical carcinomas. J Clin Microbiol 1996; 34: 745-47.

20.- Zur Hausen H. Papillomaviruses and cancer: from basic studies to clinical application. Nat Rev Cancer 2002; 2: 342-50.

21.- Schmitt M, Dondog B, Waterboer T, Pawlita M. Homogeneous amplification of genital human alpha papillomaviruses by PCR using novel broad-spectrum GP5+ and GP6+ primers. J Clin Microbiol 2008; 46 (3): 1050-9.

22.- Clifford G, Smith J S, Plummer M, Muñoz N, Franceschi S. Human papillomavirus types in invasive cervical cancer worldwide: a metaanalysis. Br J Cancer 2003; 88: 63-73.

23.- Thomas D B, Ray R M, Koetsawang A, Kiviat N, Kuypers J, Qin Q, et al. Human papillomaviruses and cervical cancer in Bangkok. I. Risk factors for invasive cervical carcinomas with human papillomavirus types 16 and 18 DNA. Am J Epidemiol 2001; 153: 723-31.

24.- Franceschi S, Plummer M, Clifford G, de Sanjosé S, Bosch X, Herrero R, et al;
International Agency for Research on Cancer Multicentric Cervical Cancer Study Groups; International Agency for Research on Cancer Human Papillomavirus Prevalence Surveys Study Group. Differences in the risk of cervical cancer and human papillomavirus infection by education level. Br J Cancer 2009; 101(5): 865-70.

25.- de Sanjosé S, Bosch X, Muñoz N, Tafur L, Gili M, Izarzugaza I, et al. Socioeconomic differences in cervical cancer: two case-control studies in Colombia and Spain. Am J Public Health 1996; 86: 1532-8.

26.- Roa J C, García P, Gómez J, Fernández W, Gaete F, Espinoza A, et al. HPV genotyping from invasive cervical cancer in Chile. Int $\mathrm{J}$ Gynaecol Obstet 2009; 105: 150-3.

27.- Larrañaga O. Eficiencia y equidad en el sistema de salud chileno (Efficiency and equity in the Chilean health care system). CEPAL/GTZ Project 1997, United Nations.

28.- WHO/ICO Information Centre on Human Papilloma Virus and Cervical Cancer. [Access on April 25, 2008]. Available at. http://www.who. int/hpvcentre/en/.

29.- Ferreccio C, Michael K, Waterboer T, Ojeda J M, Domínguez A, Franceschi S, et al. HPV antibodies in a population-based cohort of women. Chile 2001-2006. P-29.20 Abstract Book 25th International Papillomavirus Conference. May 8 - 142009 Malmö, Sweden.

30.- Ferreccio C, Domínguez A, Olcay F, Puschel K, Corvalán A, Ampuero S, et al. Dynamics of HRHPV in a population-based sample of Chilean women. P-29.34 P-29.20 Abstract Book 25th International Papillomavirus Conference. May 8 - 142009 Malmö, Sweden.

31.- Mansoura M, Toukaa M, Hasana U, Bellopedea A, Smeta A, Accardia R, et al. E7 properties of mucosal human papillomavirus types 26, 53 and 66 correlate with their intermediate risk for cervical cancer development. Virology 2007; 367: 1-9.

32.- Melo A, Montenegro S, Hooper T, Capurro I, Roa J C, Roa I. Human papillomavirus (HPV) typing in preneoplastic and neoplastic lesions of the uterine cervix in the IX Region-Chile. Rev Méd Chile 2003; 131: 1382-90.

33.- Muñoz N, Bosch F X, de Sanjosé S, Herrero R, Castellsagué X, Shah K V, et. al. International Agency for Research on Cancer Multicenter Cervical Cancer Study Group. Epidemiologic classification of human papillomavirus types associated with cervical cancer. N Engl J Med 2003; 348: 518-27.

34.- Pretet J L, Jacquard A C, Carcopino X, Charlot J F, Bouhour D, Kantelip B, et al. Human papillomavirus (HPV) genotype distribution in invasive cervical cancers in France: EDITH study. Int J Cancer 2008; 122: 428-32.

35.- Vermeulen C F, Jordanova E S, Szuhai K, Kolkman-Uljee S, Vrede M A, Peters A A, et al. Physical status of multiple human papillomavirus genotypes in flow-sorted cervical cancer cells. Cancer Genet Cytogenet 2007; 175: 132-7.

36.- Khair M M, Mzibri M E, Mhand R A, Benider A, Benchekroun N, Fahime E M, et al. Molecular detection and genotyping of human papillomavirus in cervical carcinoma biopsies in an area of high incidence of cancer from Moroccan women. J Med Virol 2009; 81: 678-84.

37.- Sandri M T, Riggio D, Salvatici M, Passerini R, Zorzino L, Boveri S, et al. Typing of human papillomavirus in women with cervical lesions: prevalence and distribution of different genotypes. J Med Virol 2009; 81: 271-7. 